\title{
Crystal Structure of Enzyme I of the Phosphoenolpyruvate Sugar Phosphotransferase System in the Dephosphorylated State*
}

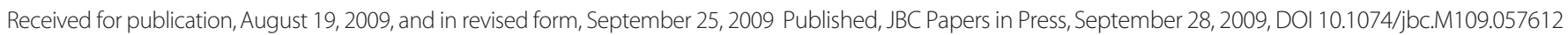

\author{
Anselm E. Oberholzer, Philipp Schneider, Christian Siebold ' , Ulrich Baumann ${ }^{2}$, and Bernhard Erni ${ }^{3}$ \\ From the Departement für Chemie und Biochemie, Universität Bern, Freiestrasse 3, CH-3012 Bern, Switzerland
}

The bacterial phosphoenolpyruvate (PEP) sugar phosphotransferase system mediates sugar uptake and controls the carbon metabolism in response to carbohydrate availability. Enzyme I (EI), the first component of the phosphotransferase system, consists of an $\mathrm{N}$-terminal protein binding domain (EIN) and a C-terminal PEP binding domain (EIC). EI transfers phosphate from PEP by double displacement via a histidine residue on EIN to the general phosphoryl carrier protein HPr. Here we report the 2.4 $\AA$ crystal structure of the homodimeric EI from Staphylococcus aureus. EIN consists of the helical hairpin HPr binding subdomain and the phosphorylatable $\beta \alpha$ phospho-histidine (P-His) domain. EIC folds into an $(\boldsymbol{\beta} \alpha)_{8}$ barrel. The dimer interface of EIC buries $1833 \AA^{2}$ of accessible surface per monomer and contains two $\mathrm{Ca}^{2+}$ binding sites per dimer. The structures of the S. aureus and Escherichia coli EI domains (Teplyakov, A., Lim, K., Zhu, P. P., Kapadia, G., Chen, C. C., Schwartz, J., Howard, A., Reddy, P. T., Peterkofsky, A., and Herzberg, O. (2006) Proc. Natl. Acad. Sci. U.S.A. 103, 16218-16223) are very similar. The orientation of the domains relative to each other, however, is different. In the present structure the $\mathrm{P}$-His domain is docked to the HPr binding domain in an orientation appropriate for in-line transfer of the phosphate to the active site histidine of the acceptor $\mathrm{HPr}$. In the $E$. coli structure the phospho-His of the $\mathrm{P}$-His domain projects into the PEP binding site of EIC. In the $S$. aureus structure the crystallographic temperature factors are lower for the HPr binding domain in contact with the $\mathrm{P}$-His domain and higher for EIC. In the E. coli structure it is the reverse.

The bacterial phosphoenolpyruvate sugar phosphotransferase system (PTS) ${ }^{4}$ mediates sugar uptake and controls the

* This work was supported by the Swiss National Science Foundation Grant 3100A0-105247.

The atomic coordinates and structure factors (code 2wad) have been deposited in the Protein Data Bank, Research Collaboratory for Structural Bioinformatics, Rutgers University, New Brunswick, NJ (http://www.rcsb.org/).

The nucleotide sequence reported in this paper has been submitted to the Swiss Protein Database under Swiss-Prot accession no. P51183.

${ }^{1}$ Present address: Division of Structural Biology, University of Oxford, Henry Wellcome Building of Genomic Medicine, Roosevelt Drive, Oxford OX3 7BN, United Kingdom.

${ }^{2}$ To whom correspondence may should be addressed. Tel.: 41-31-6314320; Fax: 41-31-6314887; E-mail: ulrich.baumann@ibc.unibe.ch.

${ }^{3}$ To whom correspondence may should be addressed. Tel.: 41-31-6314346; Fax: 41-31-6314887; E-mail: erni@ibc.unibe.ch.

${ }^{4}$ The abbreviations used are: PTS, phosphotransferase system; PEP, phosphoenolpyruvate; $\mathrm{HPr}$, histidine containing phosphocarrier protein of the PTS; El, enzyme I of the PTS; EIN, N-terminal domain of El (comprises HPr binding and P-His (sub)domains); EIC, C-terminal PEP binding domain of El; P-His domain, phosphohistidine domain of El; Ec, E. coli; Sa, S. aureus; Ell, carbon metabolism in response to carbohydrate availability. It consists of four functional phosphoprotein units that sequentially transfer phosphoryl groups from phosphoenolpyruvate (PEP) to the transported sugars (for reviews, see Refs. 1 and 2). The transport of PTS sugars is driven by the tight coupling of solute translocation with solute phosphorylation (group translocation). The control functions rely on the ratio of the phosphorylated to the dephosphorylated state of certain PTS proteins. This ratio reflects the difference between supply of phosphate by PEP and availability of solutes that consume phosphate during their uptake. The ratio increases in the absence of sugar, and it decreases when PTS substrates are taken up and/or the PEP supply is reduced.

The four PTS units which occur as either subunits or domains are phosphorylated at histidine or cysteine residues. Enzyme I (EI) is the first protein at the top of the phosphotransferase cascade. It transfers phosphate from PEP to HPr, the general high energy phosphate carrier protein of the PTS. HPr then phosphorylates the different PTS sugar transporters (EII, enzymes II) at the bottom of the cascade (Scheme I).

$$
\mathrm{PEP} \rightarrow \mathrm{EI} \rightarrow \mathrm{HPr} \rightarrow \mathrm{EIIA}^{\text {sugar }} \rightarrow \mathrm{EIIB}^{\text {sugar }} \stackrel{\text { EllC }^{\text {sugar }}}{\longrightarrow} \text { sugar }
$$

\section{SCHEME 1}

EIIs can be grouped into four structurally diverse (super)families ( 3 , 4), which have in common that they consist of three functional units, namely the phosphate transferring EIIA and EIIB and the solutetranslocating, membrane-spanning EIIC subunit/domain $(5,6)$. PTS of variable size with respect to the number EIIs (between none and more than 20 ) occur in $70 \%$ of all eubacteria (3) and in some archaebacteria (7) but not in eukaryotes.

EI of the PTS is one of the best conserved bacterial proteins with only minimal similarity to animal proteins (for review, see Ref. 8). It is a homodimer of $64-\mathrm{kDa}$ subunits that consists of an $\mathrm{N}$ - and a C-terminal domain (EIN and EIC). The x-ray structures of full-length EI of Escherichia coli and Staphylococcus carnosus $(9,10)$, of the EIN domain from E. coli (11), and of EIC from Thermoanaerobacter tengcongensis (12) have been solved. Also known is the solution structure of the complex between EIN and HPr (13-15). EIN consists of the $\alpha$-helical HPr binding subdomain that provides the docking site for HPr and the $\alpha / \beta$ P-His subdomain with its phosphoryl-acceptor histidine (His191). The P-His domain carries the phosphate from PEP bound

sugar-specific transporters of the PTS; PPDK, pyruvate phosphate dikinase; AcK, acetate kinase; MR, molecular replacement. 
to EIC to the active site His of HPr. The P-His domain has also been designated as a swiveling domain $(10,16)$. EIC is a compact eight-stranded $(\beta / \alpha)_{8}$ barrel. It contains the PEP binding site $(17,18)$ and provides the dimerization interface of EI. The PEP binding domain EIC and the P-His subdomain of EIN exhibit 30\% sequence identity with the respective domains of the pyruvate phosphate dikinase (PPDK, Swiss-Prot accession no. P22983 and PDB codes $1 \mathrm{kbl}$ and 2r82 (16, 19-22), whereas the four-helix-bundle HPr binding domain of EIN is unrelated to the nucleotide binding domain of PPDK.

In vitro experiments indicated that a phosphoryl group can be transferred to EI not only from PEP but also from ATP and acetyl phosphate through the phosphorylated intermediate of acetate kinase (AcK) and that phosphoryl transfer between AcK and Enzyme I is reversible $(23,24)$.

Equilibrium and rate constants of the EI monomer dimer transition vary strongly with protein and substrate concentrations, $\mathrm{pH}$, temperature, and other in vitro parameters. The $K_{a}$ decreases with decreasing temperature (cold sensitivity) and increasing ionic strength (ionic interactions). $K_{a}$ increases in the presence of $\mathrm{Mg}^{2+}$ and PEP $(25,26)$. The phosphoryl group is transferred from PEP to the $\mathrm{P}$-His domain of the same protein subunit, and intersubunit transfer does not occur $(10,27)$. Nevertheless, only dimeric EI can be autophosphorylated, whereas phosphate is exchanged between HPr and the monomeric EIN domain (28). It has been suggested that EI activity controls overall PTS sugar transport (29) and that EI activity itself is controlled by the monomer-dimer equilibrium. It is not clear yet which physiological signal, for instance which allosteric effector, could shift the monomer-dimer equilibrium in vivo and, thus, regulate EI activity (26) and how the apolar dimerization interface of EIC is protected against denaturation/aggregation, for instance by heterodimerization with an as yet unknown protein. It is noteworthy that the compact C-terminal dimerization domain is thermally and proteolytically unstable in full-length EI of E. coli as well as when expressed separately, whereas the monomeric, highly flexible EIN domain is resistant (30).

Here we present the X-ray structure of EI from Staphylococcus aureus (SaEI, Swiss-Prot accession no. P51183) at $2.4 \AA$ resolution. The structures of the $S a \mathrm{EI}$ and the $E$. coli $\mathrm{EI}(E c \mathrm{EI})$ (sub)domains are very similar. Their orientations relative to each other, however, are different. In the E. coli structure the $\mathrm{P}$-His domain is oriented toward EIC thus that the phosphorus of the phosphohistidine and the oxygens of the PEP analog oxalate come to within 3-4 A distance. In the present structure the $\mathrm{P}$-His domain is oriented in the alternative conformation, namely docked to the HPr binding domain with its active site His perfectly oriented for an in-line transfer of the phosphate to the acceptor HPr.

\section{EXPERIMENTAL PROCEDURES}

Expression, Purification, and Crystallization-Plasmids encoding $\mathrm{SaEI}(\mathrm{H} 191 \mathrm{~A} / \mathrm{C} 365 \mathrm{~S})$ were constructed as described previously (17). SaEI(H191A/C365S) was expressed in E. coli TH074( $\Delta p t s \mathrm{I})$, which carries a deletion of codons 131-258 of ptsI (31). Cells were grown in Luria-Bertani broth at $37^{\circ} \mathrm{C}$ to an $A_{550}$ of 0.8 and induced by $0.1 \mathrm{~mm}$ isopropyl- $\beta$-thiogalactoside. After $2.5 \mathrm{~h}$ of induction cells were harvested, resuspended in buffer A (20 mm Tris- $\mathrm{HCl}, \mathrm{pH}$ 8.5, 1 mM EDTA, 2 mM dithio-
TABLE 1

Data collection and refinement statistics for enzyme I of S. aureus

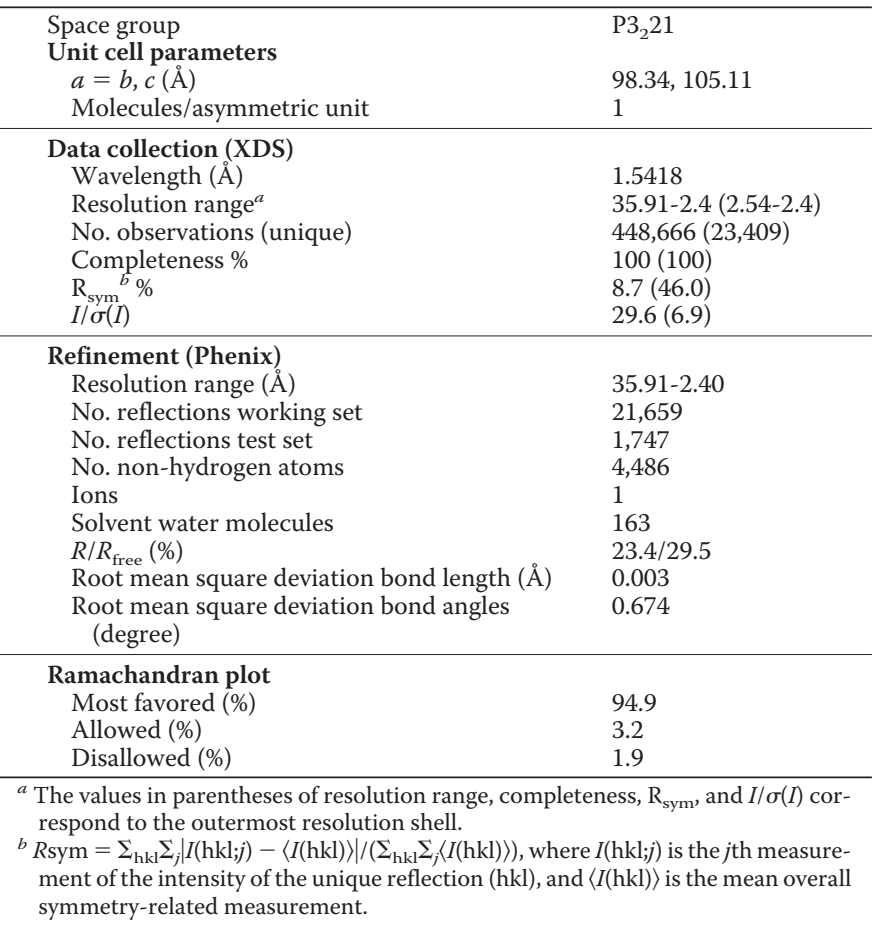

threitol), and disrupted with a French pressure cell. Insoluble material and membranes were removed by centrifugation (60 min, $150,000 \times g)$. The supernatant was applied on a High Q column (Bio-Rad) and eluted with a linear gradient of $0-600$ $\mathrm{mm} \mathrm{NaCl}$. The EI-containing fractions were pooled, dialyzed against buffer A, applied on a Resource Q (Amersham Biosciences) column, and eluted with a $\mathrm{NaCl}$ gradient. EI was further purified by gel filtration using a Superdex 200 (Amersham Biosciences) column equilibrated in buffer B $(20 \mathrm{~mm}$ Tris- $\mathrm{HCl}$, pH 8.0, $150 \mathrm{~mm} \mathrm{NaCl}, 2 \mathrm{~mm}$ dithiothreitol, $20 \mathrm{~mm} \mathrm{CaCl}_{2}$ ). The peak fractions were pooled, dialyzed against buffer $\mathrm{C}(5 \mathrm{~mm}$ Tris- $\mathrm{HCl} \mathrm{pH} \mathrm{7.5,} 2 \mathrm{~mm}$ dithiothreitol, $20 \mathrm{mM} \mathrm{CaCl}_{2}$ ), and concentrated in a Centriprep-50 concentrator to $27 \mathrm{mg}$ of protein $/ \mathrm{ml}$.

$22.3 \mu \mathrm{l}$ of SaEI was mixed with $7.6 \mu \mathrm{l}$ of buffer $\mathrm{C}$ to a final protein concentration of $20 \mathrm{mg} / \mathrm{ml}$, added to $10 \mu \mathrm{l}$ of crystallization buffer containing $0.2 \mathrm{M} \mathrm{KCl,} 20 \%$ (w/v) polyethylene glycol 3350 , and preincubated at $32.5^{\circ} \mathrm{C}$ for $5 \mathrm{~min}$. Crystals were grown at $20^{\circ} \mathrm{C}$ by vapor diffusion in hanging drops using $4 \mu \mathrm{l}$ of the preincubated solution. Crystals $\left(\sim 200 \times 200 \times 200-\mu \mathrm{m}^{3}\right.$ size) were cryo-protected by the addition of $15 \%(v / v) 2$-methyl-2,4-pentanediol and flash-cooled in a nitrogen stream at 110 K. Diffraction data to 2.4 A resolution were collected on a RaxisIV image plate area detector mounted on a Rigaku RU300 rotating-anode generator operating at $100 \mathrm{~mA}$ and $50 \mathrm{kV}$. Data were integrated and scaled with XDS $(32,33)$. Data collection statistics are reported in Table 1.

Structure Determination-SaEI crystallized in space group $\mathrm{P}_{2} 21$ with one molecule in the asymmetric unit. The structure was solved by molecular replacement (MR) using the program PHASER (34) employing the EcEI P-His domain residues (2-21, 151-232) (PDB code 2hwg) (10), the EI HPr binding 
domain residues (36-144) from S. carnosus (PDB code $2 \mathrm{hro}$ ) (9), and the EIC from T. tengcongensis (PDB code 2bg5) (12) as search models. An initial model could be built manually into the resulting electron density. The model was completed by iterative cycles of restrained refinement and model building using COOT (35) and PHENIX (36). The final model includes residues $2-571$ of $572.94 .9 \%$ of the residues are in the most favored, $3.2 \%$ are in the allowed, and $1.9 \%$ are in the disallowed region of the Ramachandran plot. Refinement statistics are given in Table 1. Figs. were prepared using PYMOL.

\section{RESULTS AND DISCUSSION}

Expression and Crystallization-Because the EIs of E.coli and $T$. tengcongensis did not afford diffraction quality crystals, EIs from seven other species were expressed in E. coli and purified for crystal screening. EI from Bacillus subtilis, Bacillus stearothermophilus, Borrelia burgdorferi, Enterococcus faecalis, Haemophilus influenzae, and S. aureus could be expressed in soluble form. They complemented glucose phosphotransferase activity of E. coli TH074 (ptsI $I^{-}$) and displayed from 4 to $7 \%$ of E. coli EI phosphotransferase activity in the heterologous in vitro phosphotransferase assay with E. coli PTS protein subunits (37). The recombinant EI domain of the E. coli multidomain protein $\mathrm{EI}^{\mathrm{Ntr}}$ (ptsP, codons 171-748) formed inclusion bodies, and the EI domain of the Pseudomonas aeruginosa multidomain protein FruB (residues 301-956) was not expressed. After optimization, EI from $S$. aureus $(\mathrm{SaEI})$ afforded the best crystals diffracting to 4.0 Å resolution (37). The same $\mathrm{SaEI}$ with an $\mathrm{N}$-terminal histidine tag did not afford $\mathrm{x}$-ray quality crystals.

To further improve the protein homogeneity of $\mathrm{SaEI}$ (judged by symmetry and sharpness of the gel filtration peak), two nonconserved cysteines predicted to be at the protein surface, Cys365 and Cys-557 of the C-terminal domain, were mutated to Ser and Ala alone and in combination (Fig. 1). Of eight mutants, the C365S mutation improved crystal quality to a diffraction of $3.5 \AA$. In addition to Cys-365, the phosphorylatable His-191 was mutated to alanine to avoid sample heterogeneity due to partial EI autophosphorylation. The double mutant EI(H191A/C365S) eventually afforded crystals that diffracted to $2.4 \AA$ resolution.

Structure Determination-Crystals of SaEI(H191A/C365S) belonged to the trigonal space group $\mathrm{P}_{2} 21(a=b=98.34, c=$ 105.11 ) with one monomer in the asymmetric unit. Only a few crystals diffracting up to $2.4 \AA$ could be obtained, whereas the majority of crystals grown in the same conditions diffracted poorly. The structure was determined by MR. A solution for the EIC domain could be easily determined by PHASER with EIC of T. tengcongensis (PDB code 2bg5) as the search model. The structure of the N-terminal domain, however, could be found neither with the model of the recombinant EIN (PDB code 1zym (11)) nor with EIN of the full-length EI (PDB code $2 \mathrm{hwg}$ (10)), probably because of the intrinsic mobility of the P-His subdomain relative to the HPr binding subdomain. An initial MR solution of poor Z-scores $(<5)$ was obtained when the two subdomains (HPr binding domain of S. carnosus, PDB code 2hro; P-His domain of E. coli, PDB code $2 \mathrm{hwg}$ ) were used as independent search models. Many runs of MR using differently trimmed search probes followed by evaluation of the resulting models by several rounds of refinement and inspection of annealed omit maps were necessary to identify correct solutions for the P-His and HPr binding subdomains. The correctness of the model was confirmed by calculation of an anomalous difference Fourier map, which showed peaks higher than $4 \sigma$ above the mean for 13 ( 10 in EIN, 3 in EIC) of 21 sulfur atoms.

Residues $2-571$ could be built into the electron density (Fig. 1). Residues Lys-208, Phe-301, Lys-416, and three short stretches corresponding to residues 151-155 (hinge I of EIN), $304-308$, and 341-356 (in EIC $\beta / \alpha$ loops 2 and 3) showed poor density in the $2 F_{o}-F_{c}$ map. Only the main chain could be traced, whereas the side chains remained invisible. These residues were modeled as alanines for the structure refinement. Contrary to expectation, Cys-365, which was mutated to a serine to improve crystallization, is buried and inaccessible from the surface. Cys-365 is not conserved and is located in a loop far from the EIC-EIN interdomain interface and at a distance of 19 $\AA$ from the PEP binding site. In view of this it is unlikely that the C365S mutation has an effect on the domain conformation.

Overall Structure-The SaEI monomer model shows the three (sub)domains (Fig. 2); that is, the N-terminal HPr binding subdomain (green, residues 27-148), the P-His subdomain (yellow, residues 2-23, 150-255), which transfers the phosphoryl groups from PEP to HPr (11), and the C-terminal PEP binding domain EIC (cyan/blue, residues 260-571). The HPr binding domain consists of four helices arranged in two hairpins; the P-His domain exhibits an $\alpha / \beta$ fold, and the PEP binding domain exhibits a $(\beta \alpha)_{8}$ barrel fold. HPr binding and P-His subdomains are linked by two crossovers of the polypeptide backbone (hinge I, residues 24-37 and 145-156) and together form the EIN domain (Figs. 1 and 2). The P-His domain is linked with the PEP binding domain through a short aperiodic segment (hinge II, residues 260-271).

The N-terminal Domain - In the N-terminal domain of SaEI, the P-His and HPr binding subdomains assume the same relative orientation as in the crystal structure of the stand-alone $E c E I N$ fragment (11) and in the NMR structure of the complex between $E c E I N$ and $E c H P r(14)$. NMR dipolar coupling measurements (38) indicated that this conformation remains unchanged irrespective of whether EIN, HPr, or both are phosphorylated or not. It appears that this is a minimal energy conformation of the ternary complex between HPr, HPr binding, and P-His domains. When HPr is docked in silico to the model of $\mathrm{SaEI}$ in the orientation given by the NMR solution structure $(14,39)$ (Fig. 3C), the distance between $\mathrm{N} \epsilon 2$ of His-191 and $N \delta 1$ of His- 15 of HPr can be reduced to $3.7 \AA$. This is less than the 4.1 $\AA$ O-P-O distance of the pentacoordinate phosphate in phosphoglucomutase near the transition state (40). To determine this distance, Ala-191 (mutation to prevent autophosphorylation) was replaced in silico with a histidine, and the rotamer conformation was adjusted such that a hydrogen bond between $\mathrm{N} \delta 1$ of His-191 and $\mathrm{O} \epsilon 1$ of the invariant Glu-127 was formed (in analogy to similar motifs observed in other PTS proteins (41-43)). However, the excellent local fit notwithstanding, $\mathrm{HPr}$ could not bind to EI in exactly the conformation shown in the model (Fig. $3 C$ ) because of a clash with the flexible $\mathrm{C}$-terminal extensions the EIC $(\beta \alpha)_{8}$ barrel. This clash can be relieved by a small displacement of EIN and EIC around the hinge II between EIN and EIC (residues 260-271). 


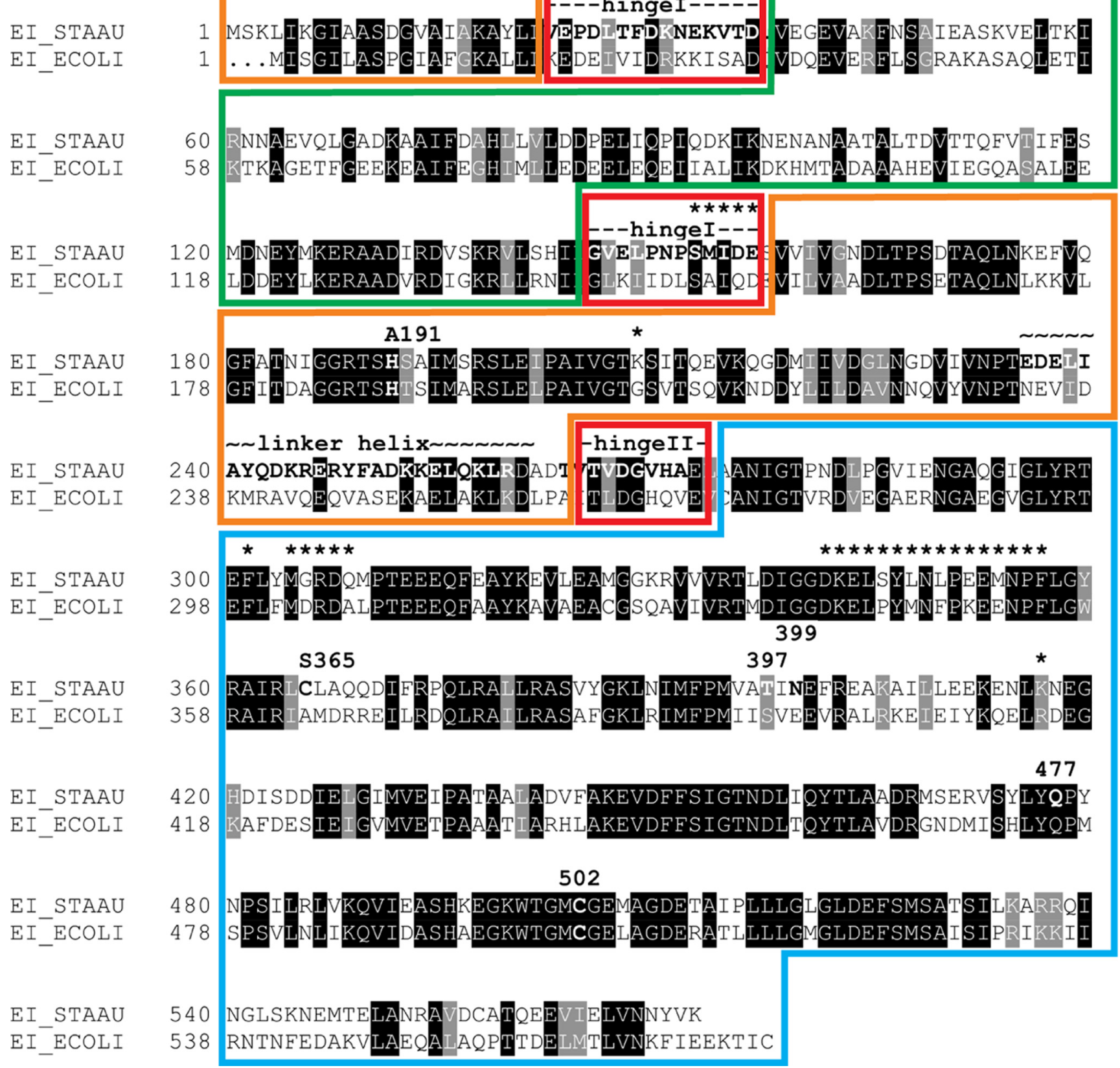

FIGURE 1. Sequence alignment of El from S. aureus (STAAU) and E. coli. The El subdomains are color coded as follows: yellow, P-His domain; green, HPr binding domain; cyan, PEP binding domain. The hinges I and II (-) are red. Note that the P-His and HPr binding domains are connected by two crossovers of the polypeptide chain (hinge I). Also indicated are the linker helix $(\sim \sim)$, the mutated residues H191A and C365S, the active site Cys-502 of the PEP binding site, the three $\mathrm{Ca}^{2+}$ binding residues of the PEP binding domain, and those residues (*) that are disordered in the $\mathrm{x}$-ray structure.

As mentioned above, it was found that EcEI was in vitro phosphorylated not only by PEP but also by AcK with either ATP or acetyl phosphate as the phosphoryl donors $(23,24)$. It was assumed that a phospho-AcK intermediate acts as phosphoryl donor toward EcEI. Such a mechanism appears unlikely today, first because AcK catalyzes the direct in-line transfer between ATP and acetate and not a double displacement with a covalent AcK-phosphate intermediate (44). Second, the P-His domain of EI and the substrate binding site of AcK lack any structural complementarity. One possibility to explain the observation is phosphorylation by Ac-P of the surface-accessible active site His-191 of the P-His domain.
Such nonenzymatic phosphorylation of active site histidines by Ac-P was observed with two-component response regulator proteins $(45,46)$.

The C-terminal Domain-The PEP binding domain SaEIC features a $(\beta \alpha)_{8}$ barrel with three extensions on the $\mathrm{C}$-terminal side of the barrel. The atomic coordinates of the monomer superimpose well with those of the crystal structure of T. tengcongensis EIC (53\% amino acid sequence identity) (12) and E. coli EIC (48\% sequence identity, Fig. 1), resulting in a root mean square deviation of $0.95 \AA$ for the paired $\mathrm{C}^{\alpha}$ atoms. Two monomers from adjacent asymmetric units form the physiological dimer (Fig. 2, $A$ and $B$ ). The contact interface between the 

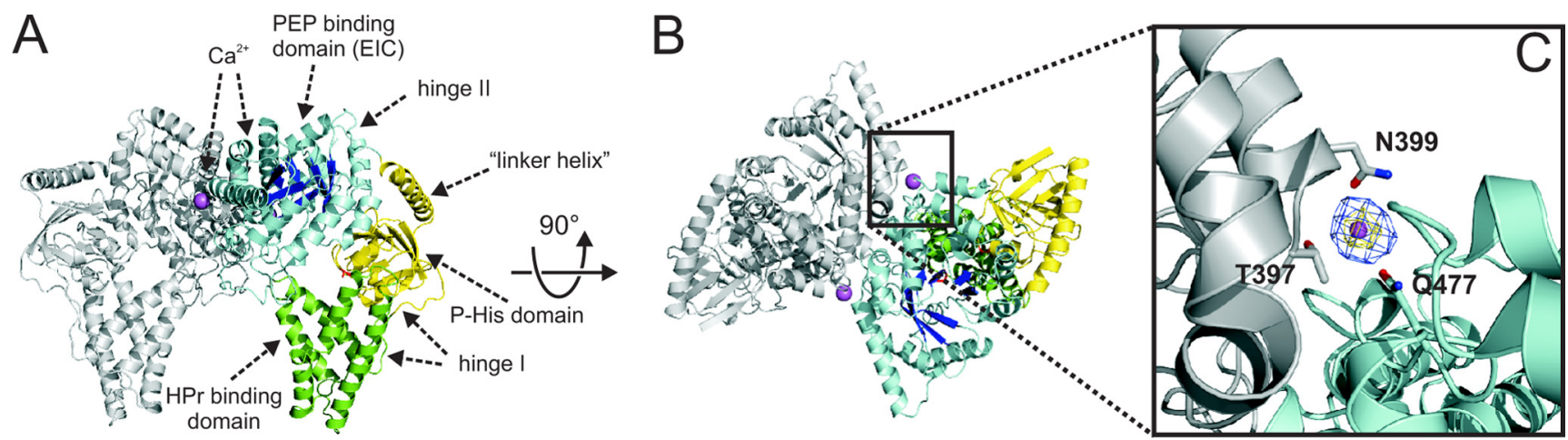

FIGURE 2. Structure of El. $A$ and $B$ show ribbon diagrams of the El dimer. The subunits are shaded in color and gray; the HPr binding domain is green, the P-His domain plus EIN-EIC linker helix is yellow, and the PEP binding domain is cyan/blue. The residues Ala-191 (H191A mutation) and Cys-504 are shown as stick models. Two $\mathrm{Ca}^{2+}$ are shown as magenta spheres. $C$, enlargement of the $\mathrm{Ca}^{2+}$ binding site is seen in the same orientation as in $B$. Atomic colors are as follows: red, oxygen; blue, nitrogen; gray, carbon. The imaginary Fourier map is depicted in chicken wire representation and contoured at 3 (blue) and 5 (yellow) S.D. above the mean. Thr-397 and Asn-399 from monomer 1 and Gln-477 from monomer 2 are shown as stick models.

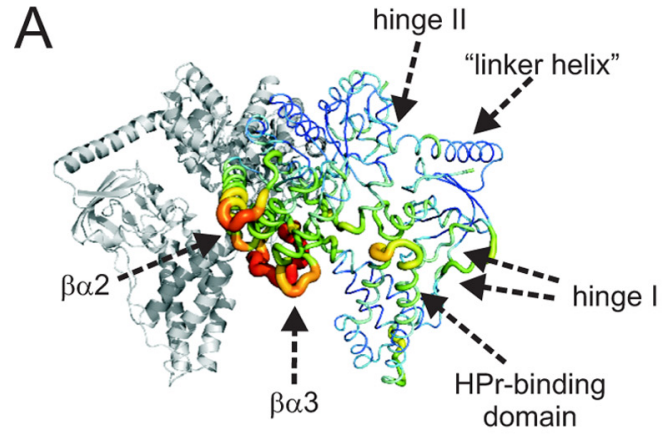

S. aureus

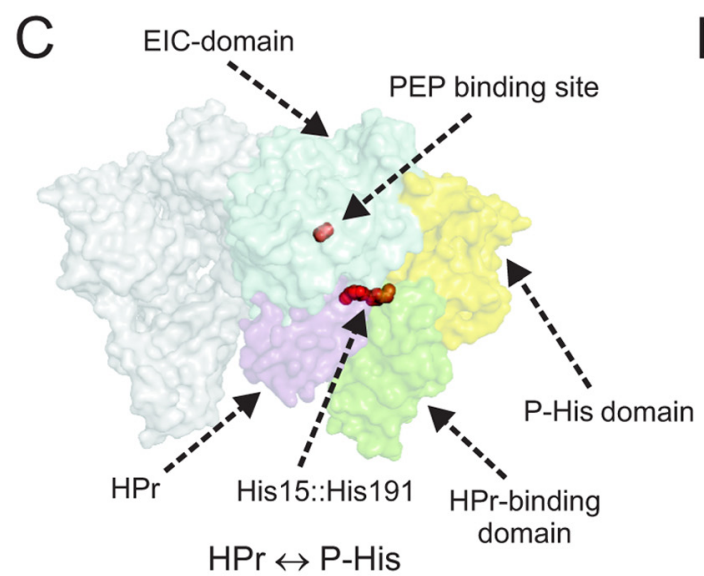

S. aureus
B

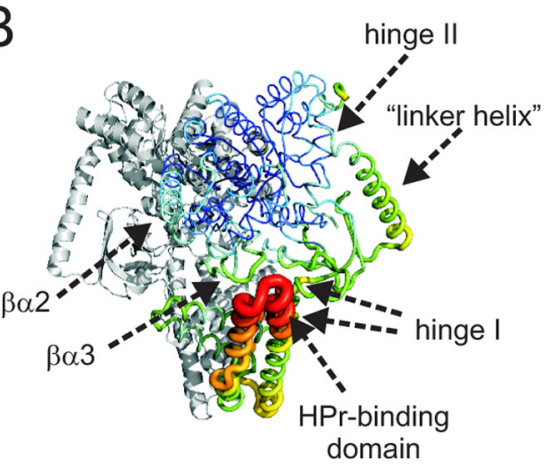

E. coli

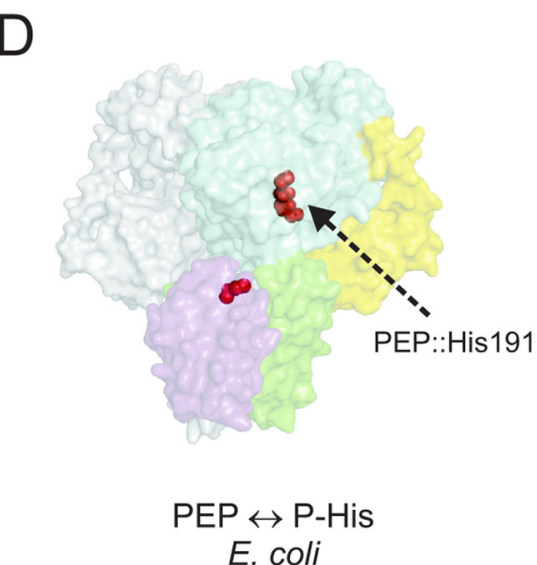

E. coli

An anomalous difference Fourier map showed two peaks above $5 \sigma$ over mean within the dimer interface of $S a$ EIC (Fig. 2C). We assigned the difference Fourier peaks to $\mathrm{Ca}^{2+}$ ions because an anomalous signal at the $\mathrm{CuK}_{\alpha} \mathrm{x}$-ray emission wavelength is expected of $\mathrm{Ca}^{2+}$ only but not of any other positively charged ion of the crystallization buffer. 20 $\mathrm{mM} \mathrm{Ca}{ }^{2+}$ was present because this concentration was beneficial for the homogeneity of EI during purification.

$\mathrm{Ca}^{2+}$ is located in an electronrich cation hole at the edge of the dimer interface where it is coordinated by the side chain oxygens of Thr-397 and Asn-399 from one and Gln-477 of the second subunit (Fig. $2 C)$. The three residues are conserved (S/T, N/Q/E, Q) but are not invariant in homologous EIs. It was shown that $\mathrm{Mg}^{2+}$ alone and $\mathrm{Mg}^{2+}$ plus PEP together shift the monomer-dimer equilibrium toward the dimeric form and stabilize $E c$ EIC by 7 and $14{ }^{\circ} \mathrm{C}$, respectively, against thermal unfolding. The stoichiometry of $\mathrm{Mg}^{2+}$ binding was estimated to be two $\mathrm{Mg}^{2+}$ per monomer with $K_{d}$ values of 0.39 and $5.8 \mathrm{~mm}(25)$. The high affinity $\mathrm{Mg}^{2+}$ occupies the PEP binding site of $E c E I C$, where it coordinates two oxygen atoms of the inhibitor oxalate and one oxygen of the His-189 phosphoryl two C-terminal domains buries $1833 \AA^{2}$ of solvent-accessible surface per monomer. Although the $\mathrm{SaEIC}$ and $T$. tengcongensis EIC protomers superimpose well, the protomer orientation in the dimer differs by a $7^{\circ}$ rotation around the normal to the crystallographic dimer axis.

group on the P-His domain (10). The low affinity $\mathrm{Mg}^{2+}$ might complex to the interfacial site, which has been identified by the $\mathrm{Ca}^{2+} \cdot \mathrm{Ca}^{2+}$ improved purification and crystallization. $\mathrm{Mg}^{2+}$ may affect other stability parameters by the same mechanism. 


\section{conformation I}

A

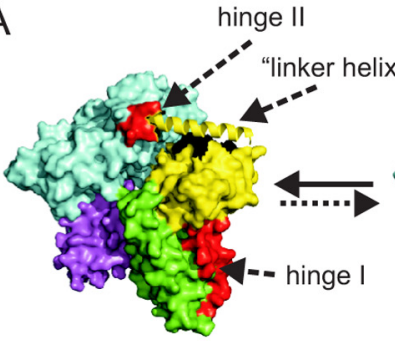

$\mathrm{HPr} \leftrightarrow \mathrm{P}-\mathrm{His}$

S. aureus

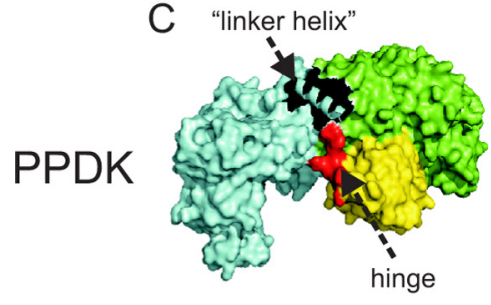

$\mathrm{P}$-His $\leftrightarrow$ NBD
$X$

conformation II

B

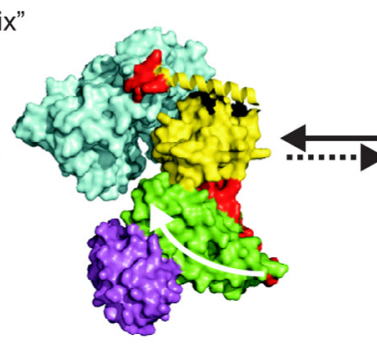

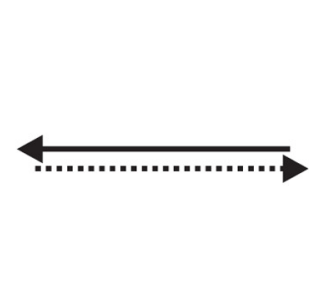

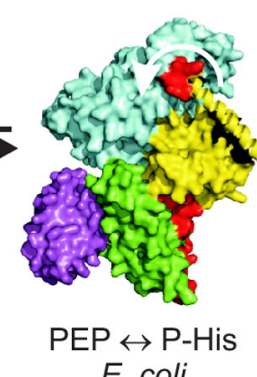

E. coli

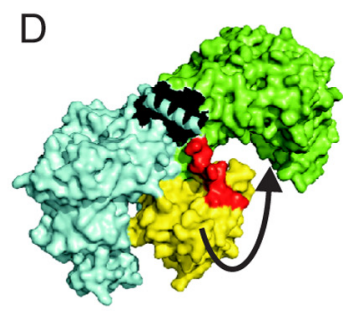

$\mathrm{PEP} \leftrightarrow \mathrm{P}$-His

FIGURE 4. Comparison of domain motions in El and PPDK. El, HPr (magenta) is docked to the HPr binding domain (green) in the conformation given by the NMR solution structure of the EIN-HPr complex (PDB code 3eza) $(14,39)$. Conformation II, shown is El with the P-His domain (yellow) projecting into the PEP binding site of EIC (cyan). $X$, hypothetical intermediate; the P-His and EIC domains have dissociated by a rotation around hinge II (red) that is located at the C-terminal end of the linker helix (schematic). In this intermediate HPr is not yet associated with the P-His domain. Conformation I, the HPr binding domain has snapped back to the P-His domain by a rotation and a bending motion of hinge I (red) located between the P-His domain and the $\mathrm{HPr}$ binding domain. PPDK, Conformation II, the P-His domain (yellow) projects into the PEP binding site (cyan). Conformation I, the P-His domain has swung to the nucleotide binding domain (NBD, green) by a rotation (swiveling motion) around the hinge (red) located at the $\mathrm{N}$-terminal end of the linker helix (schematic). Only one protomer of El and PPDK is shown.

Conformation of the EI Dimer-The P-His domain is in contact with the HPr binding domain of EIN (Fig. 2, $A$ and $B$ ). If HPr is in silico-docked to the HPr binding domain (Fig. $3 C$ ) in the orientation given by the NMR solution structure of the EINHPr complex $(14,39)$, then the ternary complex between the P-His domain, the HPr binding domain, and HPr buries $\sim 860$ $\AA^{2}$ of accessible surface area. The free energy of this interaction corresponds to an experimental complex dissociation constant of $5 \mu \mathrm{M}$ (38). For comparison, about $920 \AA^{2}$ of solvent-accessible surface are buried between the P-His and the EIC domains when the phosphorylated His-191 projects into the PEP binding site of EIC (Fig. 3, $B$ and $D$ ) (10).

A comparison of the crystallographic temperature factors between $S a E I$ and $E c E I$ shows that the factors are low for the domains in contact with the P-His domain and increased in the uncomplexed domains (Fig. 3, $A$ and $B$ ). For example, loops $\beta \alpha 2$ and $\beta \alpha 3$ of EIC are well ordered when in contact with the phosphorylated P-His domain as in EcEI (Fig. 3B) but display poor electron density without this contact as in $\mathrm{SaEI}$ (Fig. 3A). These two loops also participate in the EIC dimer interface. The free energy of the ternary contact (EIC.EIC·phospho-P-His domain) could account for the stronger protomer association of phospho-EI (26) in comparison with the binary contact (EIC.EIC) of dephosphorylated EI. The $\beta \alpha 2$ and $\beta \alpha 3$ loops of T. tengcongensis EIC are well defined even in the absence of a P-His domain. They $260-271$ ) between the P-His and the EIC domain (Fig. 4B). In the second step the HPr binding domain, which was displaced away from EIC in conformation II, snaps back toward the P-His domain to afford conformation I (Fig. 4A). Snapping involves a $60^{\circ}$ twist and a gentle bending of the hinge I (residues 24-37 and 145-156) between the P-His and the HPr binding domains (Figs. 1 and 4, $A$ and $B$ ). It is noteworthy that the $\alpha \beta$ core of the $\mathrm{P}$-His domain and the conspicuous C-terminal "linker helix" (residues 235-259, Figs. 1 and 4) move as a single rigid body. The short turn (residues 232-234) between the $\alpha \beta$ core and the linker helix does not function as a flexible hinge.

EI shares two homologous domains with the PPDK, namely the PEP binding (EIC) and the P-His domains (16). The HPr binding domain of EIN and the nucleotide binding domain of PPDK, however, have completely different folds and different locations relative to the PEP binding domain. PPDK is shown in Fig. 4, $C$ and $D$, in an orientation such that the $(\beta \alpha)_{8}$ cores of the PEP binding domains (cyan) of PPDK and EI are aligned. The HPr binding domain (green) with docked HPr (magenta) of EI is located at five o'clock above the picture plane (Fig. 4A), and the nucleotide binding domain of PPDK (green) is located at two o'clock behind the picture plane (Fig. 4C).

In EI the PEP binding domain and the HPr binding domain move relative to each other such that the distance between the phosphoryl donor PEP and the acceptor His-15 of HPr 


\section{Structure of S. aureus Enzyme I}

varies between $17 \AA$ for $\mathrm{SaEI}$ (conformation I, P-His domain projects toward His 15 of $\mathrm{HPr}$ ) and $35 \AA$ for EcEI (conformation II, P-His domain projects into the PEP binding site) (Fig. 4, $A$ and $C$ ). The P-His domain (yellow) first rotates around hinge II, and then the acceptor (complex of HPr binding domain and $\mathrm{HPr}$ ) moves around hinge I toward the P-His domain (compare Fig. 4, $B$ with $A$ ). The linker helix remains tightly associated with the $\beta / \alpha$ core of the P-His domain (yellow) of EI.

In PPDK, the PEP binding and nucleotide binding domains are coupled, and the $45 \AA$ distance between the phosphoryl donor PEP and the acceptor AMP.PP does not change (Fig. 4, $C$ and $D$ ). Only the P-His domain moves from the donor to the acceptor. The conformational changes of PPDK are described in detail by Lim et al. (22). The P-His domain of PPDK first disengages from the PEP binding site and then rotates anticlockwise around the hinge (red) (Fig. 4, from $D$ to $C$ ). The PPDK hinge is formed by two antiparallel peptide segments. The first connects the nucleotide binding domain with the $\mathrm{P}$-His domain. The second connects the $\mathrm{P}$-His domain with the $\mathrm{N}$ terminus of the linker helix (Fig. 4, $C$ and $D$ ). The linker helix of PPDK remains tightly associated with the $(\beta / \alpha)_{8}$ core of the PEP binding domain (cyan) and not with the P-His domain as in EI. Thus, only domain folds are conserved between EI and PPDK but not the structural elements that govern their dynamic interaction.

In summary, modest rigid body motions of the P-His domain relative to the EIC PEP binding domain and of the HPr binding relative to the P-His domain suffice to transfer phosphate from PEP to HPr. Compared with the compact structures of $E c \mathrm{EI}$ and $S a \mathrm{EI}$, the three (sub)domains of $S$. carnosus EI (83\% sequence identity with S. aureus) are stretched over a length of $110 \AA$ (9). The P-His domain shows weak electron density and does not contact the PEP and HPr binding domains. The open structure may represent one of many short-lived conformers as postulated in Patel et al. (26), because there is no evidence that the shortest reaction pathway (outlined in Fig. 4) also is the energetically most favorable one. Alternatively this elongated conformation may be caused by crystal lattice contacts.

Acknowledgment-The help of Priska Bähler is gratefully acknowledged.

\section{REFERENCES}

1. Görke, B., and Stülke, J. (2008) Nat. Rev. Microbiol. 6, 613-624

2. Deutscher, J., Francke, C., and Postma, P. W. (2006) Microbiol. Mol. Biol. Rev. 70, 939-1031

3. Barabote, R. D., and Saier, M. H., Jr. (2005) Microbiol. Mol. Biol. Rev. 69, $608-634$

4. Saier, M. H., Hvorup, R. N., and Barabote, R. D. (2005) Biochem. Soc. Trans. 33, 220-224

5. Robillard, G. T., and Broos, J. (1999) Biochim. Biophys. Acta 1422, 73-104

6. Siebold, C., Flükiger, K., Beutler, R., and Erni, B. (2001) FEBS Lett. 504, 104-111

7. Bolhuis, H., Palm, P., Wende, A., Falb, M., Rampp, M., Rodriguez-Valera, F., Pfeiffer, F., and Oesterhelt, D. (2006) BMC Genomics 7, 169

8. Ginsburg, A., and Peterkofsky, A. (2002) Arch. Biochem. Biophys. 397, 273-278

9. Márquez, J., Reinelt, S., Koch, B., Engelmann, R., Hengstenberg, W., and
Scheffzek, K. (2006) J. Biol. Chem. 281, 32508-32515

10. Teplyakov, A., Lim, K., Zhu, P. P., Kapadia, G., Chen, C. C., Schwartz, J., Howard, A., Reddy, P. T., Peterkofsky, A., and Herzberg, O. (2006) Proc. Natl. Acad. Sci. U.S.A. 103, 16218-16223

11. Liao, D. I., Silverton, E., Seok, Y. J., Lee, B. R., Peterkofsky, A., and Davies, D. R. (1996) Structure 4, 861-872

12. Oberholzer, A. E., Bumann, M., Schneider, P., Bächler, C., Siebold, C., Baumann, U., and Erni, B. (2005) J. Mol. Biol. 346, 521-532

13. Garrett, D. S., Seok, Y. J., Peterkofsky, A., Clore, G. M., and Gronenborn, A. M. (1997) Biochemistry 36, 4393-4398

14. Garrett, D. S., Seok, Y. J., Peterkofsky, A., Gronenborn, A. M., and Clore, G. M. (1999) Nat. Struct. Biol. 6, 166-173

15. Peterkofsky, A., Wang, G., Garrett, D. S., Lee, B. R., Seok, Y. J., and Clore, G. M. (2001) J. Mol. Microbiol. Biotechnol. 3, 347-354

16. Herzberg, O., Chen, C. C., Kapadia, G., McGuire, M., Carroll, L. J., Noh, S. J., and Dunaway-Mariano, D. (1996) Proc. Natl. Acad. Sci. U.S.A. 93, 2652-2657

17. Garcia-Alles, L. F., Flükiger, K., Hewel, J., Gutknecht, R., Siebold, C., Schürch, S., and Erni, B. (2002) J. Biol. Chem. 277, 6934-6942

18. Seok, Y. J., Lee, B. R., Gazdar, C., Svenson, I., Yadla, N., and Peterkofsky, A. (1996) Biochemistry 35, 236-242

19. Herzberg, O., Chen, C. C., Liu, S., Tempczyk, A., Howard, A., Wei, M., Ye, D., and Dunaway-Mariano, D. (2002) Biochemistry 41, 780-787

20. Wei, M., Li, Z., Ye, D., Herzberg, O., and Dunaway-Mariano, D. (2000) J. Biol. Chem. 275, 41156-41165

21. Xu, Y., Yankie, L., Shen, L., Jung, Y. S., Mariano, P. S., Dunaway-Mariano, D., and Martin, B. M. (1995) Biochemistry 34, 2181-2187

22. Lim, K., Read, R. J., Chen, C. C., Tempczyk, A., Wei, M., Ye, D., Wu, C., Dunaway-Mariano, D., and Herzberg, O. (2007) Biochemistry 46, 14845-14853

23. Fox, D. K., and Roseman, S. (1986) J. Biol. Chem. 261, 13487-13497

24. Fox, D. K., Meadow, N. D., and Roseman, S. (1986) J. Biol. Chem. 261, $13498-13503$

25. Patel, H. V., Vyas, K. A., Mattoo, R. L., Southworth, M., Perler, F. B., Comb, D., and Roseman, S. (2006) J. Biol. Chem. 281, 17579-17587

26. Patel, H. V., Vyas, K. A., Savtchenko, R., and Roseman, S. (2006) J. Biol. Chem. 281, 17570-17578

27. García-Alles, L. F., Alfonso, I., and Erni, B. (2003) Biochemistry 42, $4744-4750$

28. Seok, Y. J., Lee, B. R., Zhu, P. P., and Peterkofsky, A. (1996) Proc. Natl. Acad. Sci. U.S.A. 93, 347-351

29. Weigel, N., Kukuruzinska, M. A., Nakazawa, A., Waygood, E. B., and Roseman, S. (1982) J. Biol. Chem. 257, 14477-14491

30. LiCalsi, C., Crocenzi, T. S., Freire, E., and Roseman, S. (1991) J. Biol. Chem. 266, 19519-19527

31. Hesterkamp, T., and Erni, B. (1999) J. Mol. Microbiol. Biotechnol. 1, 309-317

32. Kabsch, W. (1993) J. Appl. Crystallogr. 26, 795-800

33. Kabsch, W. (1988) J. Appl. Crystallogr. 22, 916-924.

34. McCoy, A. J., Grosse-Kunstleve, R. W., Storoni, L. C., and Read, R. J. (2005) Acta Crystallogr. D Biol. Crystallogr. 61, $458-464$

35. Emsley, P., and Cowtan, K. (2004) Acta Crystallogr. D Biol. Crystallogr. 60, $2126-2132$

36. Adams, P. D., Grosse-Kunstleve, R. W., Hung, L. W., Ioerger, T. R., McCoy, A. J., Moriarty, N. W., Read, R. J., Sacchettini, J. C., Sauter, N. K., and Terwilliger, T. C. (2002) Acta Crystallogr. D Biol. Crystallogr. 58, $1948-1954$

37. Siebold, C. (2002) Structural Studies on Proteins of the Bacterial Phosphotransferase System. Ph.D thesis, University of Bern

38. Suh, J. Y., Cai, M., Williams, D. C., Jr., and Clore, G. M. (2006) J. Biol. Chem. 281, 8939-8949

39. Suh, J. Y., Cai, M., and Clore, G. M. (2008) J. Biol. Chem. 283, $18980-18989$

40. Lahiri, S. D., Zhang, G., Dunaway-Mariano, D., and Allen, K. N. (2003) Science 299, 2067-2071

41. Christen, S., Srinivas, A., Bähler, P., Zeller, A., Pridmore, D., Bieniossek, C., Baumann, U., and Erni, B. (2006) J. Biol. Chem. 281, 23129-23137

42. Nunn, R. S., Marković-Housley, Z., Génovésio-Taverne, J. C., Flükiger, K., 


\section{Structure of S. aureus Enzyme I}

Rizkallah, P. J., Jansonius, J. N., Schirmer, T., and Erni, B. (1996) J. Mol. Biol. 259, 502-511

43. Zurbriggen, A., Jeckelmann, J. M., Christen, S., Bieniossek, C., Baumann, U., and Erni, B. (2008) J. Biol. Chem. 283, 35789-35796

44. Gorrell, A., Lawrence, S. H., and Ferry, J. G. (2005) J. Biol. Chem. 280, 10731-10742
45. Lukat, G. S., McCleary, W. R., Stock, A. M., and Stock, J. B. (1992) Proc. Natl. Acad. Sci. U.S.A. 89, $718-722$

46. Klein, A. H., Shulla, A., Reimann, S. A., Keating, D. H., and Wolfe, A. J. (2007) J. Bacteriol. 189, 5574-5581

47. Xue, Y., Xu, Y., Liu, Y., Ma, Y., and Zhou, P. (2001) Int. J. Syst. Evol. Microbiol. 51, 1335-1341 